\title{
Memory Part 1: Overview
}

\author{
F.D. Raslau, A.P. Klein, J.L. Ulmer, V. Mathews, and L.P. Mark
}

A common layman's conception of memory is the simple storage and retrieval of learned information that often evokes comparison to a filing system. Our everyday experience of memory, however, is in fact a complicated multifactorial process that consists of both conscious and unconscious components, and depends on the integration of a variety of information from distinct functional systems, each processing different types of information by different areas of the brain (substrates), while working in a concerted fashion. ${ }^{1-4}$ In other words, memory is not a singular process. It represents an integrated network of neurologic tasks and connections. In this light, memory may evoke comparison with an orchestra composed of many different instruments, each making different sounds and responsible for different parts of the score, but when played together in the proper coordinated fashion, making an integrated musical experience that is greater than the simple sum of the individual instruments.

Memory consists of 2 broad categories (Fig 1): short- and long-term memory. ${ }^{5}$ Short-term memory is also called working memory. Long-term memory can be further divided into declarative and nondeclarative memory. These are also referred to as explicit/conscious and implicit/unconscious memory, respectively. Declarative or explicit memory consists of episodic (events) and semantic (facts) memory. Nondeclarative or implicit memory consists of priming, skill learning, and conditioning. In everyday experiences (Fig 2), declarative memory refers to things you know that you can tell others, and nondeclarative or procedural memory to things you know that you can show by doing. ${ }^{6}$ An example of episodic memory is remembering your first day of school, whereas semantic memory is knowing the capital of Canada. Nondeclarative memory manifests as knowing how to ride a bicycle (skill learning), being more likely to use a word that you recently

Received April 9, 2014; accepted after revision April 14

From the University of Kentucky (F.D.R.), Lexington, Kentucky; and Medical College of Wisconsin (A.P.K., J.L.U., V.M., L.P.M.), Milwaukee, Wisconsin.

Address correspondence to Leighton P. Mark, MD, Froedtert Hospital, Department of Radiology, Neuroradiology Section, 9200 West Wisconsin Ave, Milwaukee, WI 53226; e-mail: Imark@mcw.edu

http://dx.doi.org/10.3174/ajnr.A4059

\section{MEMORY IS NOT A UNITARY PHENOMENON}

I. Short term memory is called working memory (seconds or minutes)

\section{Long term memory}

1. Declarative memory: explicit or conscious memory.

$$
\text { a. Episodic memory (events) }
$$

b. Semantic memory (facts)

2. Nondeclarative memory: implicit or unconscious memory.

a. Priming

i. Direct Priming

ii. Indirect Priming
a. Semantic priming
b. Perceptual priming
c. Conceptual priming

b. Skill Learning

i. Motor skill

ii. Perceptual skill

iii. Cognitive skill

c. Conditioning

FIG 1. Classification of memory.

heard (priming), and salivating when you see a favorite food (conditioning).

The process of memory is dynamic with continual change over time. ${ }^{5}$ Memory traces are initially formed as a series of connections between the hippocampus and various cortical areas that are responsible for processing the particular characteristics of an event (Fig 3). Over time, with repetitive conscious retrieval of the memory and/or sleep, connections are formed between the cortical areas relevant for that memory, while the hippocampal connections become less important. Eventually, the hippocampal connections become unnecessary and the cortical connections stand alone as an integrated representation of the memory. This occurs when the memory is consolidated. Figure 4 illustrates a more concrete example of this phenomenon. A particular event (such as a birthday party) with auditory, visual, and spatial information is acquired (encoded). The hippocampus holds a unified representation of the event and the auditory information is distributed to the superior aspect of the temporal lobe, the spatial information is distributed to the parietal lobe (inferior parietal lobule), and the visual information is distributed to the occipital cortex. The memory traces are formed as connections 
between the hippocampus and these cortical areas. At this point in time, the memory has yet to be consolidated so that retrieval of the memory requires reactivation of the particular connections between the hippocampus and cortical regions. After the memory is consolidated, the cortical-to-cortical connections strengthen and the hippocampal-to-cortical connections weaken or become unnecessary. The memory is then directly accessed from the neocortex, bypassing the hippocampus. A key point is that the early unconsolidated mem- ory consists of hippocampal-to-cortical connections that are relatively rapid forming but transient, whereas the consolidated memory consists of cortical-to-cortical connections that are slow forming and long-lasting.

Théodule Armand Ribot, a noted French psychologist, first described the concept of memory consolidation in $1882 .{ }^{7} \mathrm{He}$ noted a temporal gradient to memory loss after brain damage. The tendency for recent memories to be affected to a greater extent than remote memories has become known as Ribot's law. This commonly observed phenomenon among trauma victims underscores the transient and less stable nature of recent memories that have yet to be fully consolidated, especially if the insult occurs early in the memory processing timeframe. Memories in close chronologic proximity to the traumatic event are often lost, whereas memories that are more remote tend to be less affected. The loss of memory for events that happened before an insult/injury to the brain is called retrograde amnesia. The inability to form new memories after brain injury is called anterograde amnesia. When bilateral hippocampal formation injury occurs, the ability to form new memories can be greatly impeded because of the critical

FIG 2. The everyday experience of different aspects of memory. Adapted with permission from Breedlove SM, Watson NV, Rosenzweig MR. Biological Psychology: An Introduction to Behavioral, Cognitive, and Clinical Neuroscience. 6th ed. Sunderland, Massachusetts: Sinauer Associates; 2010 .

\section{Cortical modules}
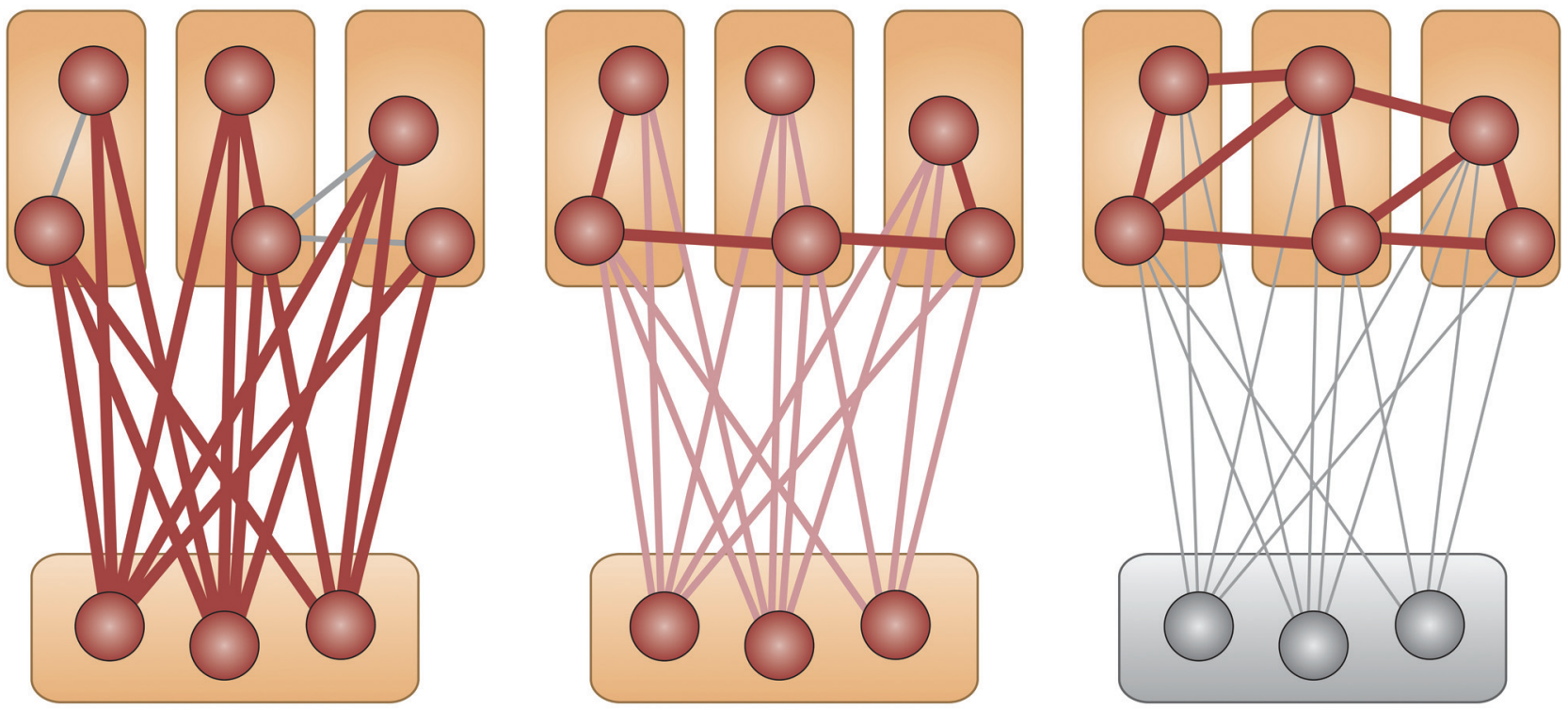

Hippocampus

Time

FIG 3. Memory consolidation. Connections between the hippocampus and various cortical modules are critical in early memory formation (left). Over time, the cortical modules form connections among themselves and the connections with the hippocampus become less important (center). When the memory is fully consolidated, only the cortical to cortical connections are important and the hippocampus becomes unnecessary (right). Adapted with permission from Frankland PW, Bontempi B. The organization of recent and remote memories. Nat Rev Neurosci 2005;6:119-30. 

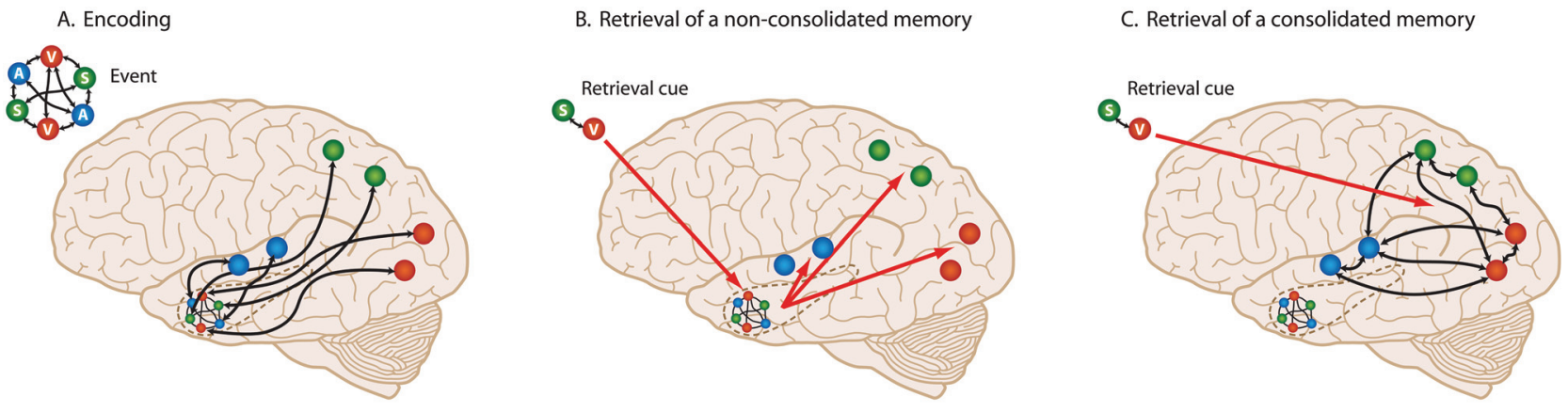

Time

FIG 4. Retrieval of nonconsolidated and consolidated memory. A, An event with audio (A), spatial (S), and visual (V) information is encoded. The hippocampus contains a unified representation of the event and forms connections with the relevant cortical areas to process the audio, spatial, and visual information. $B$, When a retrieval cue containing only spatial and visual information of the event is encountered early in the memory formation process before the memory has been consolidated, the hippocampus plays a critical role by accessing its connections with the pertinent cortical areas for the entire memory. C, After the memory is fully consolidated, the connections with the hippocampus become unnecessary and the retrieval cue accesses the memory directly from the cortical to cortical network of connections that form the unified representation of the memory. Adapted with permission from Purves D, Cabeza R, Huettel SA, et al. Principles of Cognitive Neuroscience, 2nd ed. Sunderland, Massachusetts: Sinauer Associates; 2013.

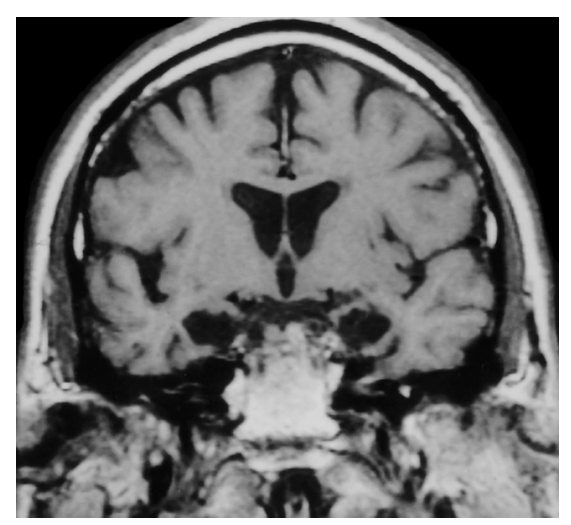

FIG 5. Patient H.M. and medial temporal lobe damage. A 1992 coronal T-weighted image of patient H.M. shows abnormal hippocampal formation hypointense signal after previous bilateral medial temporal lobe resection performed decades earlier for intractable seizures. After the surgery, the patient experienced anterograde amnesia and has been extensively studied for years afterward. Adapted from Corkin S, Amaral DG, González RG, et al. H.M.'s medial temporal lobe lesion: findings from magnetic resonance imaging.J Neurosci 1997;17:3964-79.

role the hippocampal formation plays in the early formation of memories. Figure 5 is a coronal T1-weighted image from an MR examination performed in 1992 showing bilateral medial temporal lobe injury to the extensively studied patient, H.M., who in 1953 underwent bilateral resection of the entire piriform-amygdaloid-hippocampal complex for seizures and subsequently experienced severe anterograde amnesia. ${ }^{8-11}$

Many components of the brain (prefrontal cortex, temporal lobe, parietal lobe, occipital lobe, medial diencephalon) are involved in the processes that form memories; however, this review will focus more on the medial temporal lobe and fornix. These medial temporal lobe components consist of the hippocampal formation, parahippocampal gyrus, parahippocampal cortex, entorrhinal cortex, and perirhinal cortex. The contribution of these structures to the function of memory will be the subject of the next article in the Functional Vignettes series.

\section{REFERENCES}

1. Zola-Morgan S, Squire LR. Neuroanatomy of memory. Annu Rev Neurosci 1993;16:547-63

2. Yancey SW, Phelps EA. Functional neuroimaging and episodic memory: a perspective. J Clin Exp Neuropsychol 2001;23:32-48

3. Tulving E. What is episodic memory? Curr Dir Psychol Sci 1993;2: $67-70$

4. Squire LR. Memory systems of the brain: a brief history and current perspective. Neurobiol Learn Mem 2004;82:171-77

5. Frankland PW, Bontempi B. The organization of recent and remote memories. Nat Rev Neurosci 2005;6:119-30

6. Breedlove SM, Watson NV, Rosenzweig MR. Biological Psychology: An Introduction to Behavioral, Cognitive, and Clinical Neuroscience. 6th ed. Sunderland, Massachusetts: Sinauer Associates; 2010

7. Ribot TA. Diseases of Memory: An Essay in the Positive Psychology. The International Scientific Series. New York: D. Appleton and Co; 1882

8. Corkin S, Amaral DG, González RG, et al. H.M.'s medial temporal lobe lesion: findings from magnetic resonance imaging. J Neurosci 1997;17:3964-79

9. Salat DH, van der Kouwe AJ, Tuch DS, et al. Neuroimaging H.M.: 10-year follow-up examination. Hippocampus 2006;16:936-45

10. Schmolck H, Kensinger EA, Corkin S, et al. Semantic knowledge in patient H.M. and other patients with bilateral medial and lateral temporal lobe lesions. Hippocampus 2002;12:520-33

11. Kensinger EA, Ullman MT, Corkin S. Bilateral medial temporal lobe damage does not affect lexical or grammatical processing: evidence from amnesic patient H.M. Hippocampus 2001;11:347-60 